\title{
Management of an open abdomen complicated by a high output entero-atmosferic fistula after a gastric by-pass
}

\author{
Lorena Hierro-Olabarria Salgado
}

\section{CASE REPORT}

\begin{abstract}
There is a wide spectrum of indications for negative pressure wound therapy (NPWT) including enterocutaneous fistulas, open abdomen management, abdominal wound dehiscence, open fractures, amputation wounds, sternal wound infections, vascular bypass site infection and many others. The article shows a case report of a patient with an enteroatmospheric fistula managed with NPWT. Control of intestinal contents from an entero-atmospheric fistula with the NPWT minimizes the damage to the healing bed of granulation tissue until the definitive closure of the fistula can be undertaken six to twelve months later.

After a laparoscopic gastric bypass and 5 emergency interventions because of leaks at the jejunojenunal anastomosis and complications of the abdominal wall, an entero-atmospheric fistula appeared. This fistula was managed with the NPWT during two months. In the described case, the application of negative pressure dressing system allowed the management of the entero-atmospheric fistula until it became a stoma.
\end{abstract}

Keywords-gastric bypass, jejunojejunal anastomosis leak, abdominal wall closure, laparostoma, enteroatmospheric fistula, NPWT

\section{INTRODUCTION}

$\mathbf{E}$ NTERO-ATMOSFERIC fistulas occur in the midst of an open abdomen with no overlying soft tissue. These are one of the most difficult complications of "damage control" laparotomy and are a source of significant morbidity and mortality ${ }^{1}$ The main goal in managing external fistulas is to control the enteric contents at the source of the fistula to protect the skin from corrosive effects of the enteric or pancreatic fluids contents, and to facilitate nursing care of the patient ${ }^{2[3}$ Control of intestinal contents from an enteroatmospheric fistula will minimize damage to the healing bed of granulation tissue until the definitive closure of the fistula can be undertaken six to twelve months later. It may require surgical diversion to convert the gastrointestinal opening into a stoma that can thus be controlled. NPWT can also simplify management of entero-atmospheric fistula. The

Manuscript received 23.03.2017; revised 31.10.2017. This work did not receive any financial support. Authors declare no conflict of interest.

Author affiliations: Licenciada en Medicina y Cirugía Especialidad de Cirugía General y del Aparato Digestivo Facultativo Especialista de Área Sección de Cirugía de la Pared Abdominal \& CMA Hospital Universitario Basurto. OSI Bilbao-Basurto / Osakidetza Bilbao. País Vasco., (LHOS)

*Correspondence to: Lorena Hierro-Olabarria Salgado: lrnhierro@gmail.com continuous suction of the fistula output minimizes contact time between the intestinal fluid and the exposed peritoneum, allowing effective control of intestinal spillage. Care must be taken when applying a negative pressure wound dressing in the setting of exposed (but uninvolved) bowel since it can cause formation of additional fistulas, especially if fresh suture or staple lines in the bowel are exposed.4 Additional protection can be provided by placing another layer of plastic or a biologic dressing between the bowel and the negative pressure sponge.$^{5}$ In this paper, we present a complex case of an entero-atmospheric fistula, highlighting the importance of the NPWT and the different dressing options in this therapy.

\section{CASE REPORT}

A 67- year-old woman was operated on in our hospital on April 2016 for morbid obesity by the Esophagogastric surgical team; a programmed laparoscopic bypass was performed. The 8th day after surgery she presented with clinical and analytical worsening, compatible with intestinal obstruction. (Fig. 11) Computed tomography (CT) with contrast-media enhancement images revealed a dilation of the alimentary limb and a wrinkle or obstruction at the Roux-en-Y connection. Emergency surgery was immediately performed with a laparotomic approach. The findings were a wrinkle of the jejuno-jejunal anastomosis that was causing an obstruction and dilation of the alimentary limb. During manipulation, the alimentary limb was perforated. A section of the distal portion of the bilio-pancreatic limb and a new end to side anastomosis was performed. The perforation of the alimentary limb was treated with simple suture.

Five days after the patient underwent a new emergency surgery. This time the complication was a leak of both extremes of the Roux-en-Y (or jejuno-jejunal) anastomosis and also of the suture of the alimentary limb. The surgical team carried out a section of the alimentary limb from the stitches that had failed to the Roux-en-Y (distal) and a new end to end manual anastomosis leaving a $100 \mathrm{~cm}$ long remnant alimentary limb. Finally, they performed a new side to side anastomosis for the jejuno-jejunostomy, distal from the previous anastomosis (Fig. 22). The abdominal wall closure was difficult because it was under tension, so they 


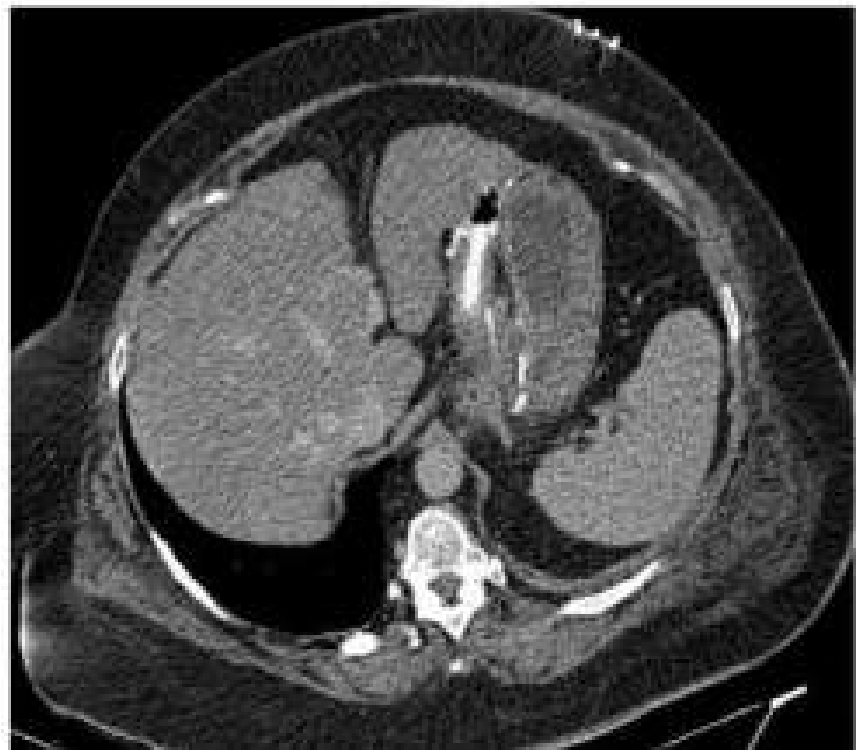

Figure 1. Computer tomography (CT) with contrast-media revealed a dilation of the alimentary limb and a wrinkle or obstruction at the Roux-en-Y connection.
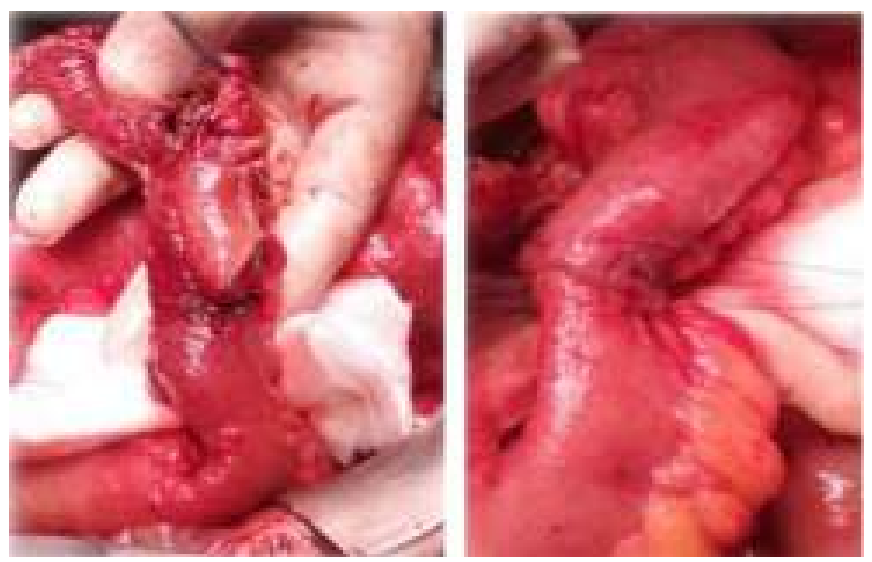

Figure 2. Leak of both extremes of the jejuno-jejunal anastomis. End to end manual anastomosis

decided to make a lateral incisions of both anterior rectus abdominis sheaths and then close the wall with Smead-Jones Polydioxanone stitches.

The abdominal closure was complicated six days later because of a subcutaneous haematoma with an active bleeding. The patient again underwent surgical treatment with another laparotomy, where a partial leak of the jejuno-jejunostomy was identified and treated with some simple stitches. Closure of the abdominal wall again was carried out, this time with " $\mathrm{X}$ " type stitches.

Two days after this closure a skin-covered evisceration took place. The decision of performing a new surgery to repair the evisceration was made. At this time, the primary closure of the abdominal wall was impossible, so the surgeons decided to put a $25 \times 15 \mathrm{~cm}$ Polyglactin mesh as a bridge. The intra-abdominal pressure in this moment was $12,3 \mathrm{mmHg}$.

A month later, the skin and subcutaneous tissue suture
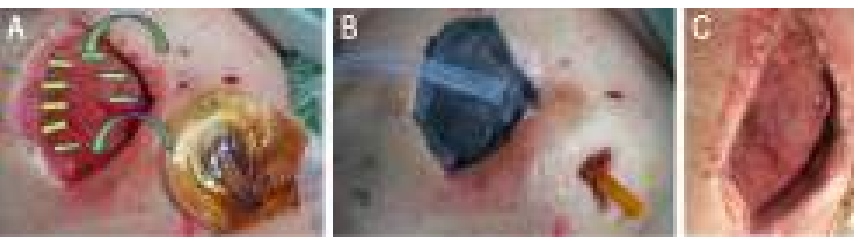

Figure 3. A: Laparostoma in midline and drainage with a stoma bag on jejunojunestomy leak. B: Placement of the NPWT dressing, C: Wound appearance after NPWT

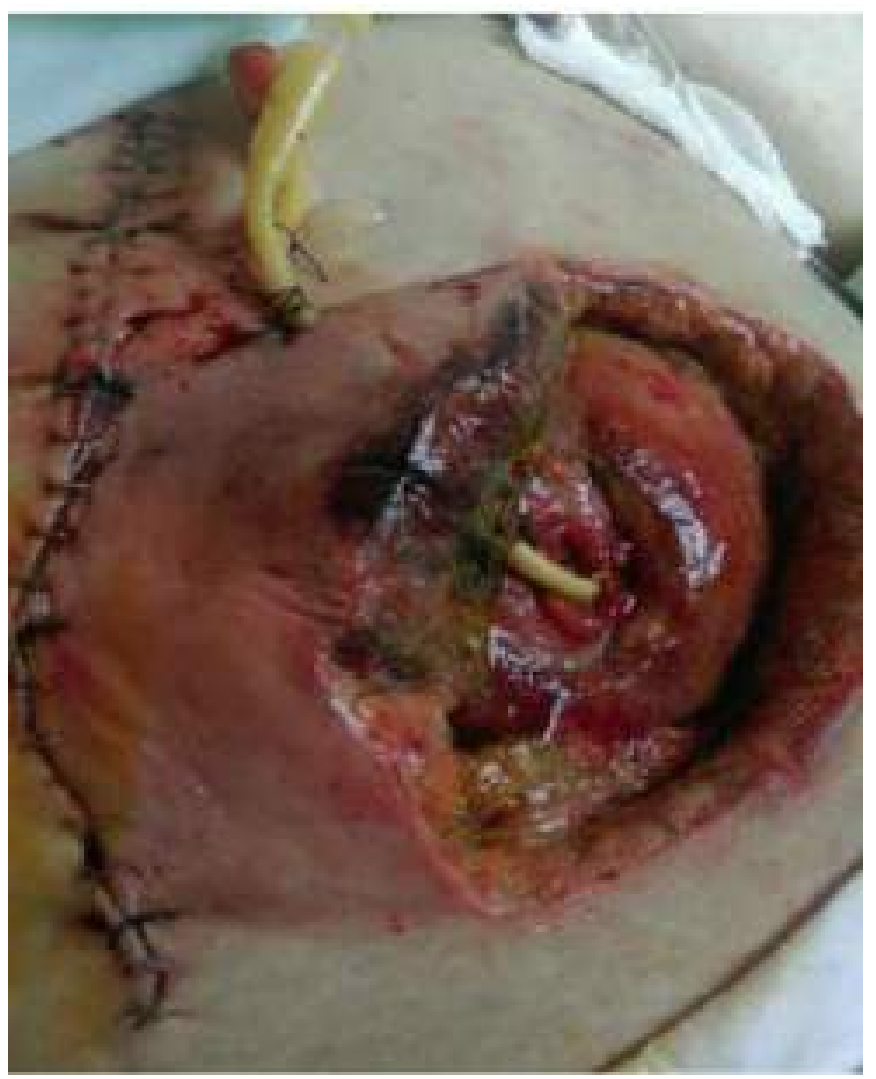

Figure 4. Entero-atmospheric fistula with the feeding catheter at the distal loop.

failed and the abdominal closure became a laparostomy with granulation tissue. In addition, the patient developed a new intra-abdominal leak that was conducted to the skin with a drain (Fig. 3).

At this moment, the patient was referenced to us. We decided to employ the NPWT (Fig. 3). Fifteen days later we decided to perform a new surgery to close the mid-line, achieve a better drainage of the leak of the jejunojejunostomy and place a feeding catheter at the distal loop (Fig. 4).

After this procedure, we faced an entero-atmospheric fistula and we considered some different options to manage it in the (Fig. 5).

\section{A. T-DRAIN}

Placing a T-drain (red color in the (Fig. 5p) into both loops of the fistula (proximal and distal). This option allows us to keep the nutrition tube in the distal loop. Then, we should place the black sponge polyurethane with an abdominal foil 

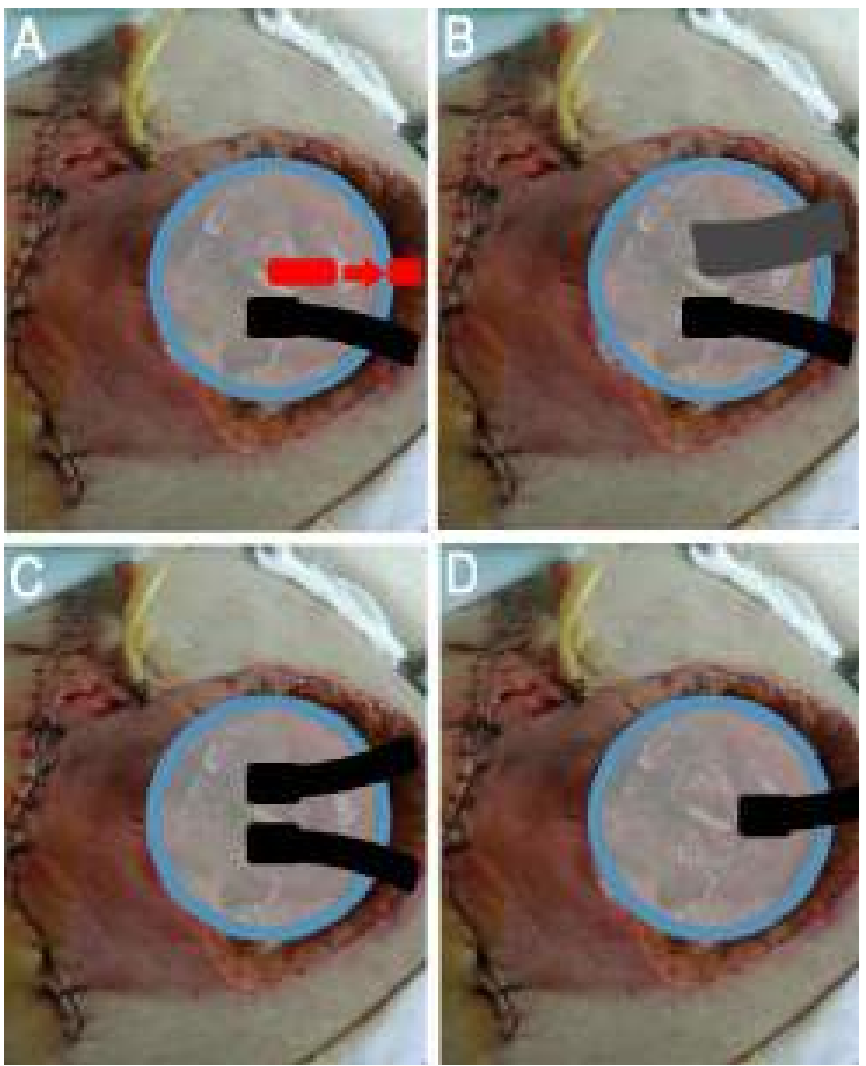

Figure 5. Surgical management options of the fistula: A: T-drain and NPWT. B: Stoma bag and NPWT C: Two NPWT ports. D: NPWT port directly on the fistula

below (on the intestines surface). The T-drain should go through the sponge and we should put some stoma paste around the sponge (blue color at the (Fig. 5), especially in the lowest part and in damaged skin. Finally, we should place the adhesive on the surface (the drain needs to go through it) and cut the hole in the adhesive for the port (preferably in the lowest part of dressing). We should connect the T-drain to the normal suction with low pressure (too high pressure may increase the secretion and may also lead to the closure of the drain if it is elastic) and the port into NPWT system (Fig. 5).

\section{B. STOMA BAG}

Placing the abdominal foil on the surface of intestines with a hole above the fistula. After placing the black sponge on the wound, surrounded by the stoma paste, cut an opening in the sponge right above the fistula (the diameter should be not too small, 6-7 cm), and put stoma paste around it. We should cut the hole in the foil above the fistula, with a diameter of about 3-4 cm. Cut the hole for NPWT drain. Use a stoma bag above the fistula opening and connect the NPWT port (Fig. 5).

\section{TWO NPWT PORTS}

It consists of placing the abdominal foil on the surface of the intestines with a hole above the fistula. A ring of stoma
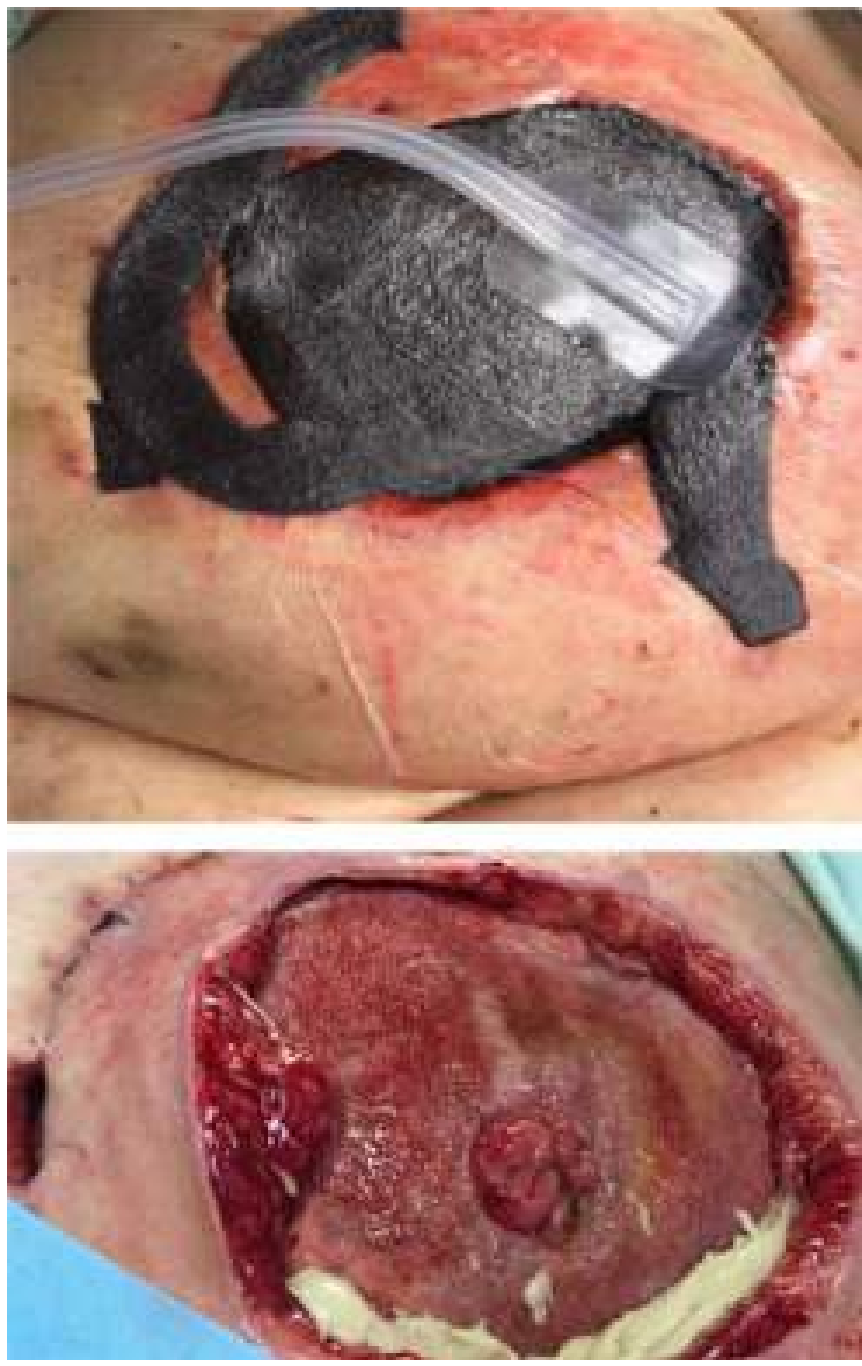

Figure 6. The NPWT dressing with the port directly on the fistula.

paste should be placed above the fistula, leaving an opening around it, minimum $5 \mathrm{~cm}$ of width. We should place the black sponge with stoma paste around it. It is necessary to make the sponge thinner above the fistula. We should cut two openings for the NPWT ports and connect them with NPWT. The lowest port (on the wound surface) should have a negative pressure around $125 \mathrm{mmHg}$. After $3-6$ days we should try to change pressures: the lowest above the fistula and the highest applied to the tissue around (Fig. 5).

\section{NPWT PORT DIRECTLY ON THE FISTULA}

We chose this option. We first placed the abdominal foil on the surface of the intestines with a hole above the fistula, with a ring of stoma paste (leaving an opening around it, minimum $5 \mathrm{~cm}$ width). Then we placed the polyurethane sponge with a hole above the fistula with a ring of stoma paste. Finally, we put the adhesive foil on the surface with a hole above the fistula and we connected the port of the NPWT system in this hole (Fig. 6)(Fig. 7). On the one hand, the patient required a change of the dressing every 12-24 hours being it very complex. Initially, We did not achieve the management of 

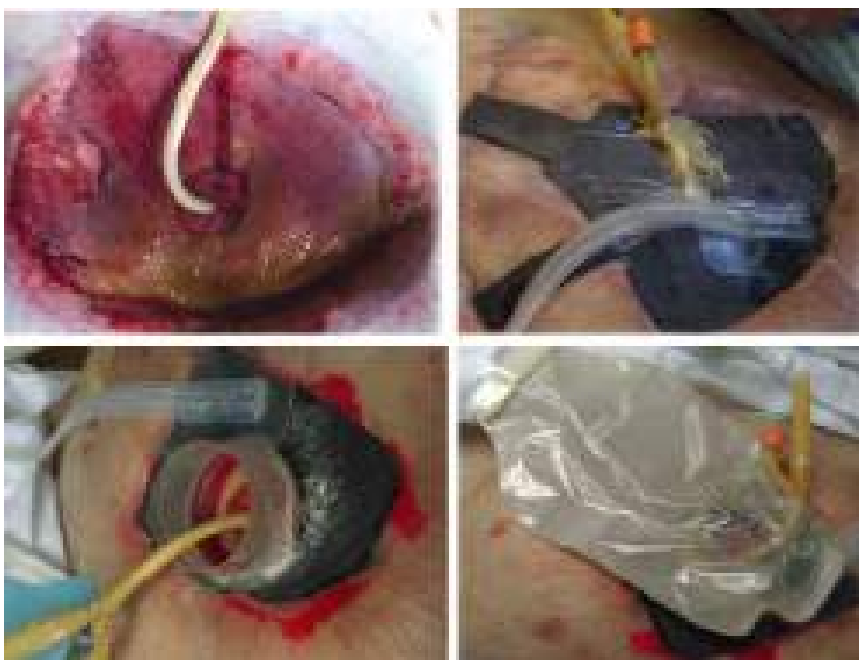

Figure 7. The NPWT dressing adapted with the catheter for feeding and the urine jar as a collector.
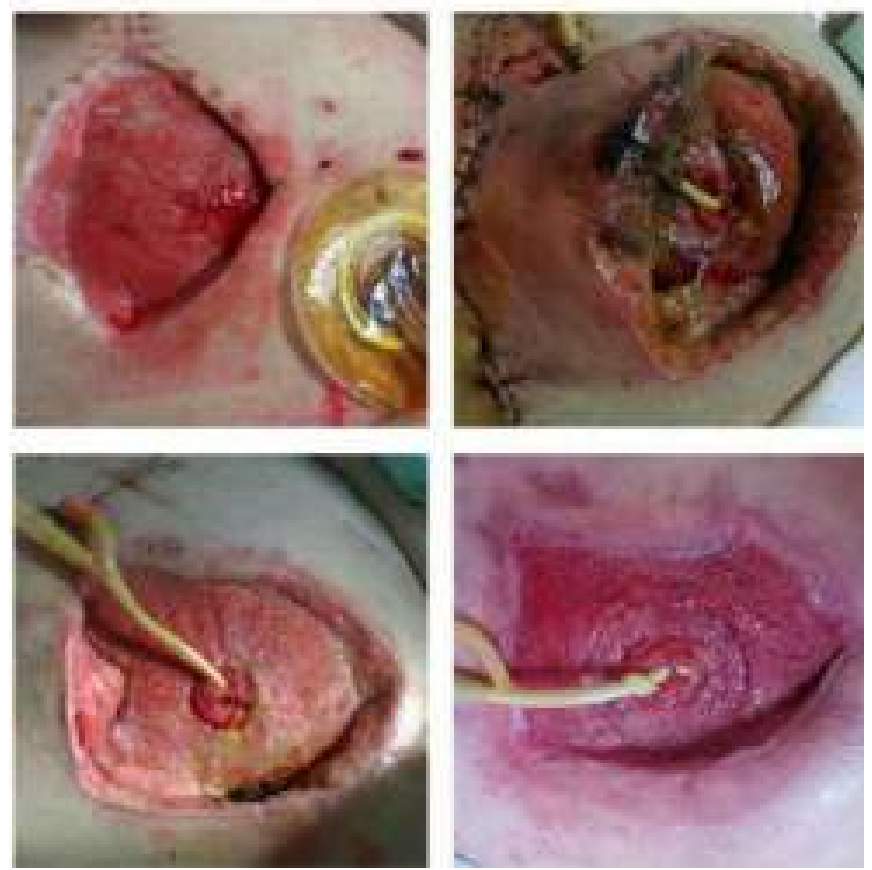

Figure 8. The evolution from the laparostoma to the entero-atmospheric fistula and the improvement of the granulation tissue almost becoming a stoma around the fistula.

the intestinal fluids, the bile accumulated on the healing bed of granulation tissue and was creating an important damage and skin irritation. On the other hand, it was difficult to keep the feeding catheter while we were applying this kind of dressing (Fig. 77). In order to prevent the accumulation of the bile, we placed an urine jar as a collector, but this accessory made the dressing more complex (Fig. 8). We can see the notorious improvement using the NPWT during two months (four months since the first surgery)(Fig. 9p. At this moment we had achieved the goal of managing the laparostoma and of the entero-atmospheric fistula, but we could not ensure a continuous nutritional support for the patient.
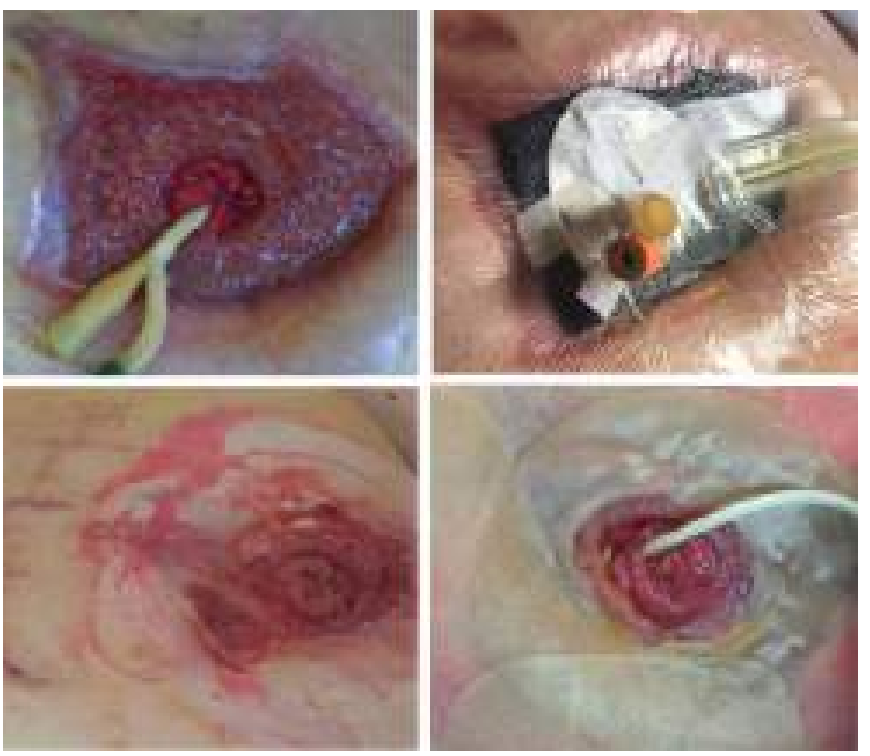

Figure 9. Last days of NPWT.Pictures of the dressing after NPWT.

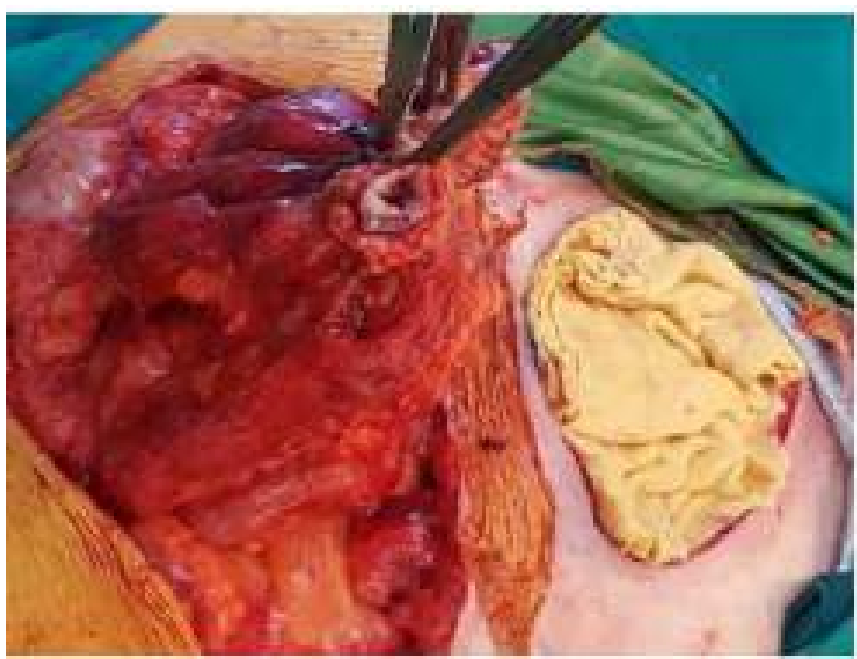

Figure 10. Adhesions between the graft and the underlying bowel.

In this situation, even though it is known that the indication of the definitive closure of the fistula should be made after 6-12 months of its appearance (in order to reduce the risk of intestinal injury), we decided to perform an operation to restore the gastrointestinal continuity. It was long time that the patient was feeding with the parenteral nutrition (with the subsequents hepatic alterations), because we could not get an enteral feeding with a catheter at the distal loop: at the beginning, the reason was the negative pressure that made the enteral feeding go out from the wound, and later, the difficult control of the infusion of enteral feeding with the bomb. Because all of these reasons we decided to come ahead of time with the surgery. We found adhesions between bowels and between the graft and the underlying bowel (Fig. 10). The three intestinal limbs of the Roux-en-Y-anastomosis were adhered to the abdominal wall, so we performed a section of each one $(5 \mathrm{~cm})$ followed by a new manual anastomosis. The alimentary limb became $1 \mathrm{~m}$ long, the biliary limb 40 


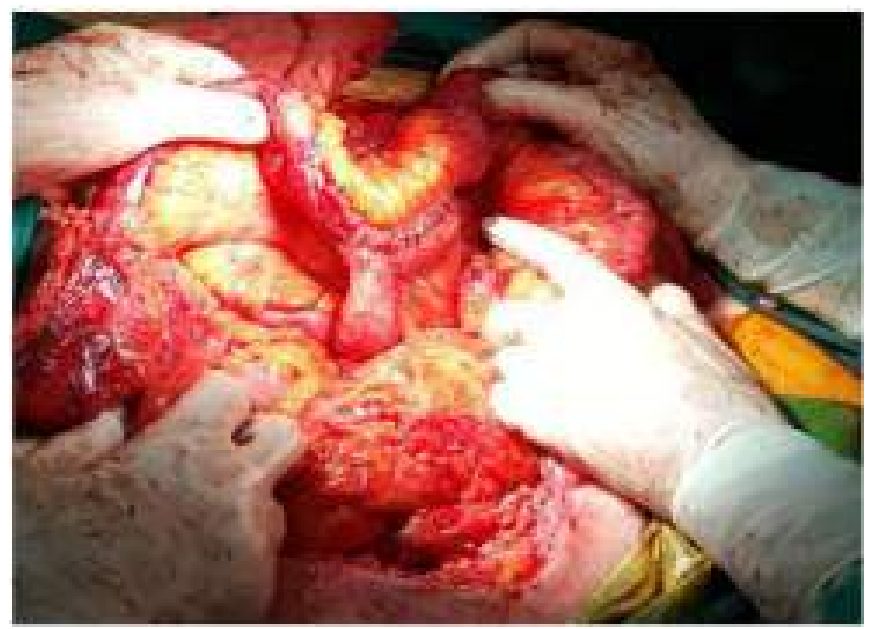

Figure 11. Picture of the three intestinal limbs with adhesions to the abdominal wall on the fistula. We sectioned these adhesions before performing the section of the three limbs

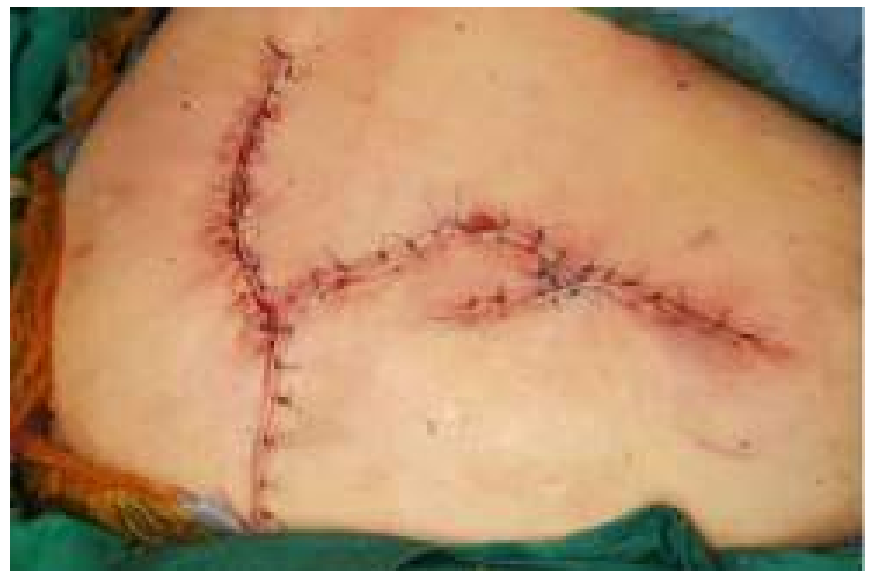

Figure 12. Result after the surgery.

$\mathrm{cm}$ and the distal limb $1.40 \mathrm{~m}$ long (Fig. 11). Once the fistula is resected and gastrointestinal continuity restored, the abdomen should be closed using standard techniques, provided this will not result in undue tension. We carried out a skin subcutaneous tissue approximation with advancement flaps (Fig. 12.

\section{DISCUSSION}

Vacuum-assisted closure (VAC), sometimes referred to as microdeformational wound therapy (MDWT) or negative pressure wound therapy (NPWT), has revolutionized wound care over the last 15 years. When using NPWT devices significant shrinkage of the wound can be observed as the wound edges come together by a combination of foam shrinkage and drawing the edges of the wound together by foam contact. At the wound interface, the foam creates microdeformations that stretch cells and activate molecular pathways for angiogenesis and cell division. For wounds with oedema, these devices have the capacity to remove a large amount of fluid

NPWT can be very useful for the management of enteroatmospheric fistula ${ }^{7}$ Continuous suction of the fistula output minimizes contact time between intestinal fluid and exposed peritoneum, thus effectively controlling intestinal spillage. Patients with entero-atmospheric fistula who have failed five to six weeks of nonoperative management will likely need surgery to definitively manage the fistula. Although the timing of definitive surgery to close the fistula is a matter of judgment, once it has been determined that the fistula is not likely to resolve on its own, surgery should generally not be undertaken for another few months to lessen the risk of bowel injury. ${ }^{[8}$ Imaging should be repeated to ensure that fluid collections are drained, and inflammation has resolved. Dense adhesions begin to form in the open abdominal wound after approximately one week of exposure and remain treacherous for at least six to eight weeks. The presence of a fistula is usually associated with a severe the inflammatory response that leads to dense adhesions, known as "obliterative peritonitis", that make early surgery hazardous 9010 buy and sell usd In addition to issues related to adhesion formation, definitive management of an enteroatmospheric fistula should not be considered until the skin graft is supple and can be pinched between the thumb and index finger, which signifies the existence of a plane between the graft and underlying bowel.11 This signifies that there is a tissue plane between the skin graft and the gut, facilitating one of the more difficult steps of abdominal wall reconstruction, which is dissection of the skin graft off the bowel. The aim of surgery is to eliminate the fistula, which usually requires resection of the a segment of bowel that is the origin of the fistula, reestablishment of gastrointestinal continuity, and tension-free closure of the abdomen with well-vascularized soft tissue. 12

Once the fistula is resected and gastrointestinal continuity restored, the abdomen should be closed using standard techniques, provided this will not result in undue tension. The goal of the definitive surgery in the management of entero-atmospheric fistula, besides closing the fistula, is to reconstruct the abdominal wall with durable well-vascularized tissue. A two-team approach has the advantage of providing a well-rested plastic surgeon focused on reconstruction after a long and tedious visceral dissection by the general surgery team ${ }^{12}$ Closure of large or complex abdominal wall defects associated with entero-atmospheric fistulas may require advancement flap techniques. One option for abdominal closure is the component separation technique, provided the rectus abdominis muscle remains intact ${ }^{13}$ Defects up to $10 \mathrm{~cm}$ in the upper abdomen, $20 \mathrm{~cm}$ in the mid-abdomen, and $8 \mathrm{~cm}$ in the lower abdomen can be closed using this technique. Other options may include random, pedicle, or free flaps with microvascular reconstruction.

\section{CONCLUSION}

The application of NPWT in the management of enteroatmospheric fistula is a widely accepted therapeutic method. NPWT has many beneficial clinical effects : it creates a moist environment, improves fluid removal, reduces tissue edema, contracts the wound mechanically stimulates the wound bed, induces cell proliferation, alters blood flow to the wound edges stimulates neoangiogenesis and the formation 
of granulation tissue. In our case, applying NPWT allowed turning an entero-atmospheric fistula into a stoma around the fistula. However, it is important to remember that patients with entero-atmospheric fistula who have failed five to six weeks of nonoperative management will likely need surgery to definitively manage the fistula.

\section{ACKNOWLEDGEMENT}

I would like to thank the following companies for their assistance with the collection of my data: Angel Zorraquino Gonzalez, Monica Elías Aldama, Ana Isabel Gutierrez Ferreras, Patiricia Perez de Villarreal Amilburu, Begoña Ochoa Villalabeitia.

\section{REFERENCES}

[1] S. Majercik, M. Kinikini, and T. White, "Enteroatmospheric fistula: from soup to nuts," Nutrition in Clinical Practice, vol. 27, no. 4, pp. 507-512, 2012.

[2] C. Cro, K. George, J. Donnelly, S. Irwin, and K. Gardiner, "Vacuum assisted closure system in the management of enterocutaneous fistulae," Postgraduate medical journal, vol. 78, no. 920, pp. 364-365, 2002.

[3] P. Banwell, S. Withey, and I. Holten, "The use of negative pressure topromote healing," British journal of plastic surgery, vol. 51, no. 1, p. 79, 1998.

[4] M. Rao, D. Burke, P. Finan, and P. Sagar, "The use of vacuum-assisted closure of abdominal wounds: a word of caution," Colorectal Disease, vol. 9, no. 3, pp. 266-268, 2007.
[5] R. Jamshidi and W. P. Schecter, "Biological dressings for the management of enteric fistulas in the open abdomen: a preliminary report," Archives of Surgery, vol. 142, no. 8, pp. 793-796, 2007.

[6] C. Huang, T. Leavitt, L. R. Bayer, and D. P. Orgill, "Effect of negative pressure wound therapy on wound healing," Current problems in surgery, vol. 51, no. 7, pp. 301-331, 2014.

[7] W. P. Schecter, A. Hirshberg, D. S. Chang, H. W. Harris, L. M. Napolitano, S. D. Wexner, and S. J. Dudrick, "Enteric fistulas: principles of management," Journal of the American College of Surgeons, vol. 209, no. 4, pp. 484-491, 2009.

[8] E. K. Johnson and P. L. Tushoski, "Abdominal wall reconstruction in patients with digestive tract fistulas," Clinics in colon and rectal surgery, vol. 23, no. 03, pp. 195-208, 2010.

[9] V. W. Fazio, T. Coutsoftides, and E. Steiger, "Factors influencing the outcome of treatment of small bowel cutaneous fistula," World journal of surgery, vol. 7, no. 4, pp. 481-488, 1983.

[10] G. L. Hill, "Operative strategy in the treatment of enterocutaneous fistulas," World journal of surgery, vol. 7, no. 4, pp. 495-501, 1983.

[11] T. W. Jernigan, T. C. Fabian, M. A. Croce, N. Moore, F. E. Pritchard, G. Minard, and T. K. Bee, "Staged management of giant abdominal wall defects: acute and long-term results," Annals of surgery, vol. 238 , no. 3, p. 349, 2003.

[12] W. P. Schecter, A. Hirshberg, D. S. Chang, H. W. Harris, L. M. Napolitano, S. D. Wexner, and S. J. Dudrick, "Enteric fistulas: principles of management," Journal of the American College of Surgeons, vol. 209 no. 4, pp. 484-491, 2009.

[13] K. C. Shestak, H. J. Edington, and R. R. Johnson, "The separation of anatomic components technique for the reconstruction of massive midline abdominal wall defects: anatomy, surgical technique, applications, and limitations revisited," Plastic and reconstructive surgery, vol. 105, no. 2, pp. 731-739, 2000 